\title{
Corrigendum: The adipokine Retnla modulates cholesterol homeostasis in hyperlipidemic mice
}

Mi-Ran Lee, Chae-ji Lim, You-Han Lee, Jong-Gil Park, Seong Keun Sonn, Mi-Ni Lee, In-Hyuk Jung, Se-Jin Jeong, Sejin Jeon, Myoungsook Lee, Ki Sook Oh, Young Yang, Jae Bum Kim, Hueng-Sik Choi, Woojin Jeong, Tae-Sook Jeong, Won Kee Yoon, Hyoung Chin Kim, Jae-Hoon Choi \& Goo Taeg Oh

Nature Communications 5:4410 doi: 10.1038/ncomms5410 (2014); Published 15 Jul 2014; Updated 21 Jan 2015

In the key for Fig. $4 \mathrm{~d}$ in this Article, the labels indicating the $L d l r^{-/}$and $L d l r^{-/}-/$Retnla-Tg groups were unintentionally placed next to the wrong symbol. The correct version of Fig. 4 appears below. 
a
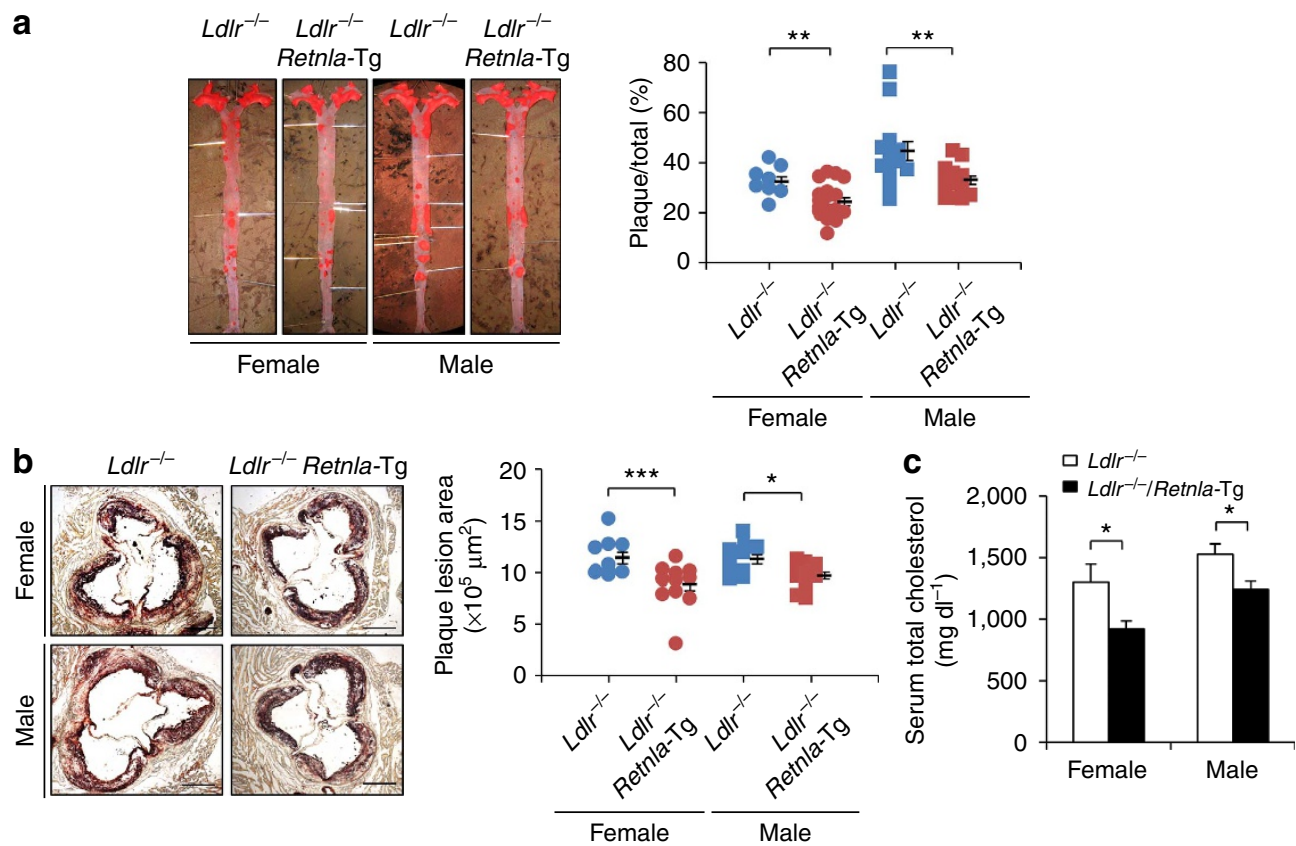

d

Female
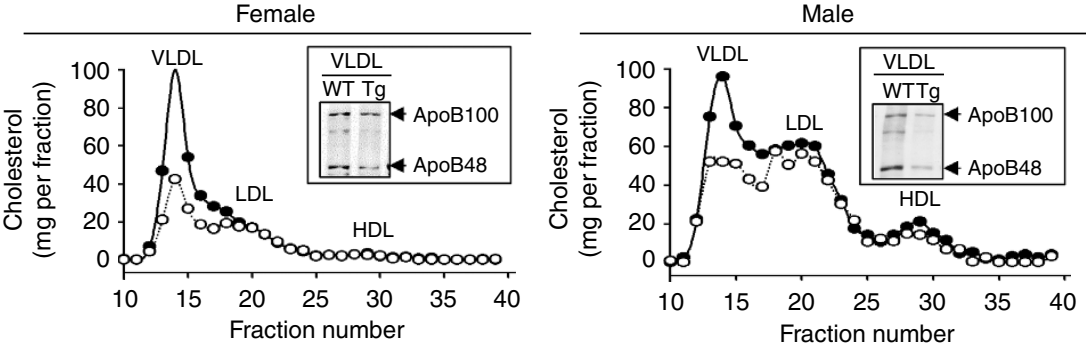

$\cdots .0 \cdots . .$. Ldlr $^{-1-/ R e t n l a-T g} \longrightarrow$ Ldlr ${ }^{-/-}$

e
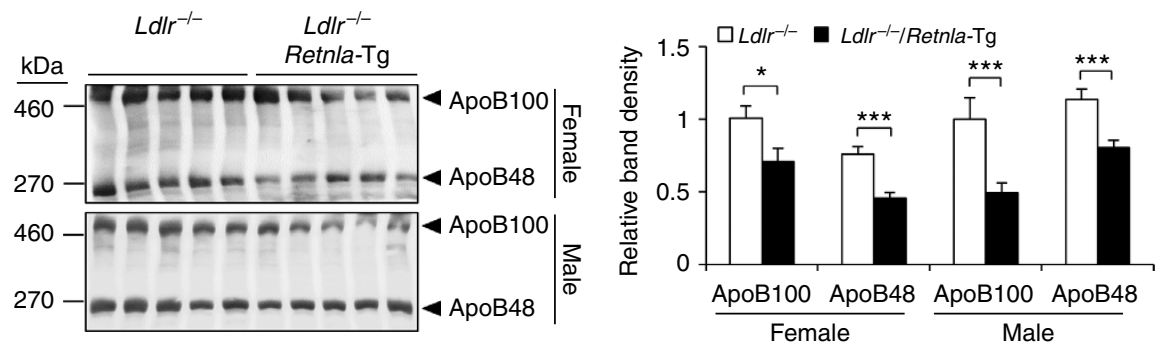

Figure 4 